\title{
The Effects of Coil Current Distribution in a Cylindrical Electron Cyclotron Resonance Reactor
}

\author{
WU Hanming, D. B. Graves ${ }^{\dagger}$, LI Ming, WANG Qin ${ }^{\ddagger}$ \\ Institute of Mechanics, Academia Sinica, Beijing 100080 \\ 'Department of Chemical Engineering, University of California Berkeley, CA 94720, USA \\ :China Central Radio and TV University, Beijing 100032
}

(Received 25 March 1994)

\begin{abstract}
Using a 2-D hybrid model, the authors have found that external currents play an important role in the plasma parameters in the reactor. The plasma density, temperature and electrostatic potential would be significantly influenced by the applied external currents.
\end{abstract}

PACS: $52.65 .+Z$

The application of electron cyclotron resonance (ECR) plasma source in microelectronics industry has been appeal to some scientists and enginecrs for many years. ${ }^{1-6}$ Some numerical simulations for ECR have been reported before. ${ }^{1,7.8}$ The most interesting plasma parameters for etching processing are plasma density, electron temperature and plasma potential. The question is how one can control these parameters by means of adjusting external conditions, i. e. coil positions and current distribution.

To find the effects of external currents, we are going to simulate two cases in which the external currents are set differently. The resonance zone shapes are primarily determined by the coil position and current distribution. In the present paper, we develop the previous work of Ref. 7. The primarily purpose of the paper is to find relation between the current and some plasma parameters.

Physical Description: In this investigation, we consider the compact plasma source as a cylindrical column with $21 \mathrm{~cm}$ length and $16 \mathrm{~cm}$ radius. The magnetic field is generated by two outside coils. The top boundary of the chamber is assumed insulated and the others grounded. Assume the argon gas be filled in the chamber with a uniform pressure $0.2 \mathrm{~Pa}$. Since the physical model is primarily the same as the Refs. 7 and 8 , the following model description is quite brief. We have assumed that electrons follow a Maxwell-Boltzmann distribution, even though we are aware that it is only an approximation. The total power input is $500 \mathrm{~W}$. The power-deposition profile is assumed as $P_{\mathrm{ECR}}=c\left[1.2-(r / R)^{4}\right] /\left[1+\left(B-B_{0}\right)^{2} /(0.0025)^{2}\right]$, where $R$ is the chamber radius, $c$ a constant determined with normalization of total power input and $B_{0}=0.095 \mathrm{~T}$ the resonance magnetic field. The ions in the model are represented as individual particles. Twenty thousands of 'superparticle' ions are followed from their point of creation as they move in the external applicd magnetic field and self-consistent clectrostatic field. Ions are followed in 2D3V. i. e. two spatial dimensions $(r, z)$ and three velocity space dimensions $\left(v_{r}, v_{\theta}, v_{z}\right)$.

Mathematical Description: The cylindrical coordinates $(r, \theta, z)$ are adopted. The geometry is supposed to be axis symmetric.

1) Electron Description: Electron is considered as a fluid. The mass conservation and energy conservation equations are written as:

(C)y the Chincse Physical Socicty 
Mass conservation:

$$
\begin{gathered}
j_{\mathrm{e}}=\mu:\left[n_{\mathrm{e}} \nabla \phi-\nabla\left(n_{\mathrm{e}} T_{\mathrm{e}}\right)\right], \\
\nabla \cdot j_{\mathrm{e}}=n_{\mathrm{e}} g\left(T_{\mathrm{e}}\right) .
\end{gathered}
$$

Energy conservation:

$$
\begin{gathered}
\boldsymbol{Q}=2 k T_{\mathrm{e}}\left(\boldsymbol{j}_{\mathrm{e}}-\boldsymbol{\mu}: n_{\mathrm{e}} \nabla T_{\mathrm{e}} / e\right), \\
\nabla \cdot \boldsymbol{Q}=n_{\mathrm{e}} P_{\mathrm{ECR}}+e \boldsymbol{j}_{\mathrm{e}} \cdot \nabla \phi-P_{\text {loss }}
\end{gathered}
$$

Poisson equation:

$$
\varepsilon_{0} \Delta \phi=e\left(n_{\mathrm{e}}-n_{\mathrm{i}}\right)
$$

where $n_{\mathrm{e}}, n_{\mathrm{i}}, \phi, T_{\mathrm{e}}, \boldsymbol{j}_{\mathrm{e}}, \boldsymbol{Q}, \mu, g$ and $\varepsilon_{0}$ are electron density, ion density, electrostatic potential, electron temperature, electron flux, electron energy flux, mobility, ionization rate and permeability, respectively. $P_{\text {loss }}=P_{\mathrm{I}}+P_{\mathrm{x}}+P_{\mathrm{M}}+P_{\mathrm{EL}}$, where $P_{\mathrm{I}}, P_{\mathrm{x}}, P_{\mathrm{M}}$ and $P_{\mathrm{EL}}$ are the power consumption for ionization, excitation, metastable excitation and elastic-neutral collision, respectively.

2) Ion Description: Ions are modeled as particles. Ion born from an ionization collision is with the background gas temperature and a Maxwellian velocity distribution. Ion-ion and electron-ion collisions are ignored in the current model. The total cross section for charge exchange cross section is: $\sigma_{\mathrm{ch}}(E)=5.1 \times 10^{-19} \mathrm{~m}^{2}[1-0.052 \ln (E)]^{2}$, where $E$ is the kinetic energy of the ion in $\mathrm{eV}$. The time step $\Delta t$ must be short so that $v_{\mathrm{ch}} \Delta t$ is small enough, where $v_{c h}$ is a charge exchange collision frequency. Otherwise, multiple collisions are likely during a single time step but will not be accounted for in the simulation. For $1 \mathrm{eV}$ ions at $0.25 \mathrm{~Pa}$, a time step of $\Delta t=1 \mu \mathrm{s}$ is acceptable. Each lost particle is counted towards the ion current to the wall. The wall absorption is supposed to be $100 \%$ and no secondary electron emission.

(3) Boundary Conditions: We assume the sheath is infinitesimally thin. The potential discontinuity between plasma sheath and wall potentials is defined as $\Delta \phi=\phi_{\mathrm{s}}-\phi_{\mathrm{w}}$. The magnitude of the sheath potential jump discontinuity determines magnitudes of electron flux, electron energy flux and ion acceleration into the wall. For collisionless sheaths, these three aspects depend on neither the sheath thickness nor the shape of the potential profile in the sheath, but only on the magnitude of $\triangle \phi$. If a Maxwellian electron velocity distribution (isotropic) is used at the plasma-sheath boundary with electron temperature $T_{\mathrm{es}}$, electron density $n_{\mathrm{cs}}$, and plasma potential $\phi_{\mathrm{s}}$, then the magnitude of the electron flux to the wall is:

$$
j_{\mathrm{ew}}=0.25 n_{\mathrm{es}} v_{\mathrm{th}} \exp \left[-e \Delta \phi /\left(k T_{\mathrm{es}}\right)\right],
$$

where $v_{\text {th }}$ is an average thermo-velocity. In an analogous way, the energy flux of the electrons is represented by

$$
Q_{\mathrm{ew}}=k T_{\mathrm{es}} j_{\mathrm{ew}}\left[2+e \Delta \phi /\left(k T_{\mathrm{es}}\right)\right]
$$

The second kind of the boundary is of dielectric wall, where the surface potential will float with respect to ground. The ion flux $j_{\text {iw }}$ is specified to determine $\phi_{\mathbf{w}}$ at each grid point of boundary, i.e. from $j_{\mathrm{iw}}=j_{\mathrm{ew}}$ and Eq. (6). Therefore, the wall potential would be

$$
\phi_{\mathrm{w}}=\phi_{\mathrm{s}}+k T_{\mathrm{e}} / e \ln \left[4 j_{\mathrm{iw}} / n_{\mathrm{es}} v_{\mathrm{th}}\right] \text {. }
$$




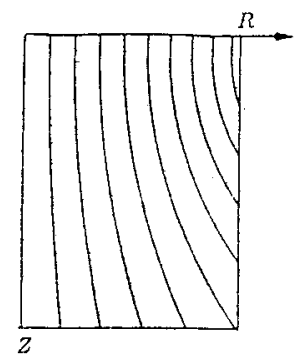

Fig. 1. Magnetic flux configuration of case $I$.

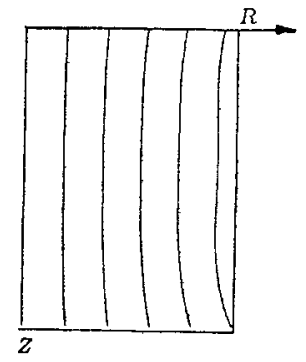

Fig. 2. Magnetic flux configuration of case II.

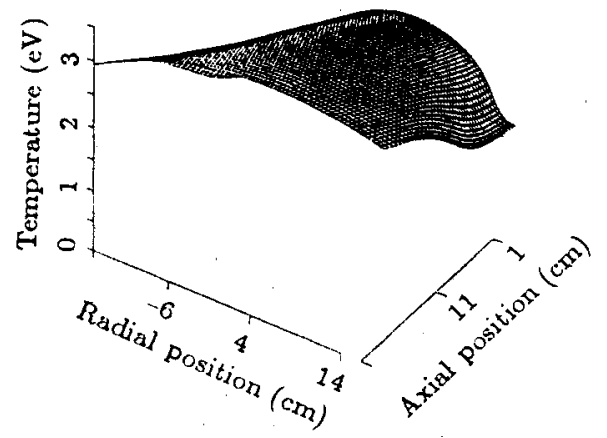

Fig. 3. Spatial distribution of electron tempcrature for case $I$.

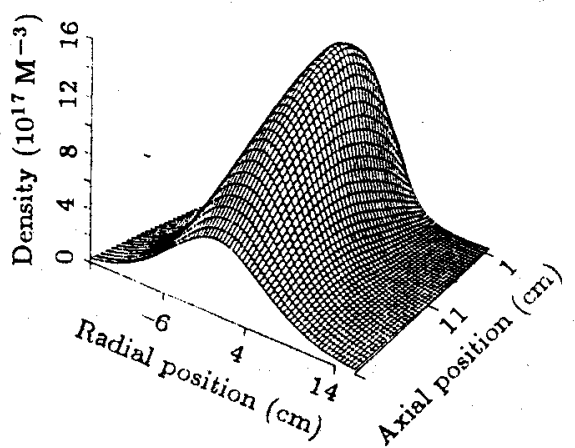

Fig. 5. Spatial distribution of plasma density for case I.
Results and Discussion: Two cases are simulated in the present paper. Each of them has its special current distribution $I=\left(I_{1}, I_{2}\right)$, where $I_{1}$ and $I_{2}$ are the currents in upper and down coils, respectively. The magnetic flux configurations generated by the external applicd currents $I=(230 \mathrm{~A}, 0)$ of case $I$ and $I=(110,110 \mathrm{~A})$ of case II are shown in Figs. 1 and 2, respectively. It is found that the radial magnetic flux component of case I is much larger than that of case II. It is obvious that, in case I, most power absorption is

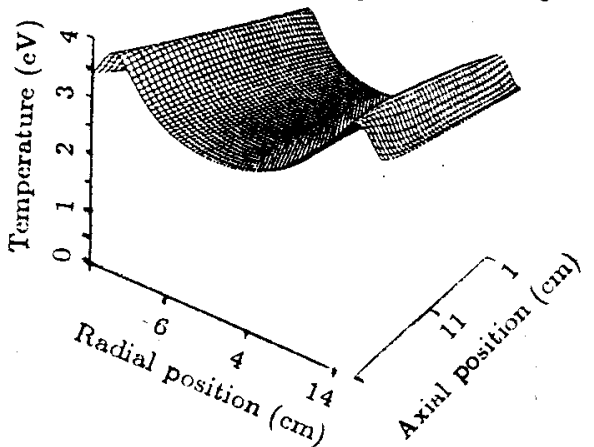

Fig. 4. Spatial distribution of electron temperature for case II.

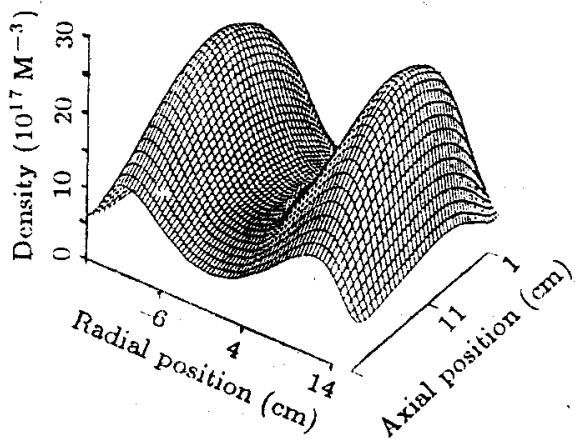

Fig. 6. Spatial distribution of plasma density for case II.

concentrated near the center, whereas, in case II, most power absorption is located near the cdge of the chamber. Geonetrically, the ECR zone shape of case I looks like an oblate ellipsoid and that of case II a donut-like. These different power deposition profiles would result in different electron temperature distributions (see Figs. 3 and 4). Since we have only consider the mobility in parallel direction, the electrons absorbed energy in the ECR area is not allowed to transfer their energy across the magnetic line. Owing a large parallel component of thermo-conductivity the electron temperature gradient along the magnetic line is small. The similar phenomenon in a compact and extended chambers were previously reported in Refs. 7 and 8 but it is not as 


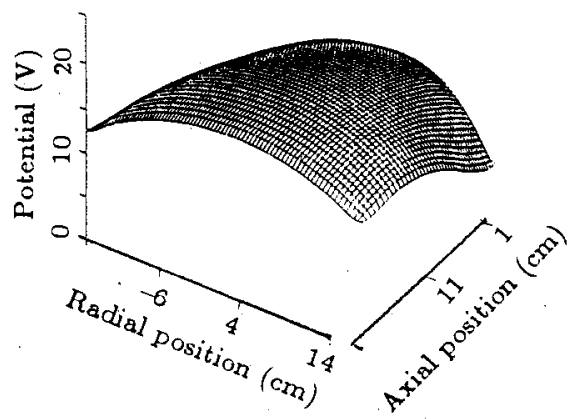

Fig. 7. Spatial distribution of plasma potential for case I.

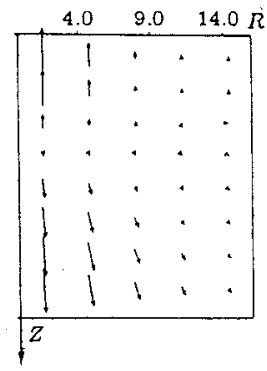

Fig. 9. Ion flux vectors in the chamber for case I.

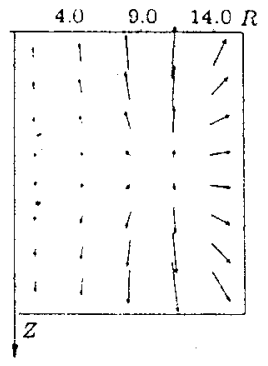

Fig. 10. Ion flux vectors in the chamber for case II.

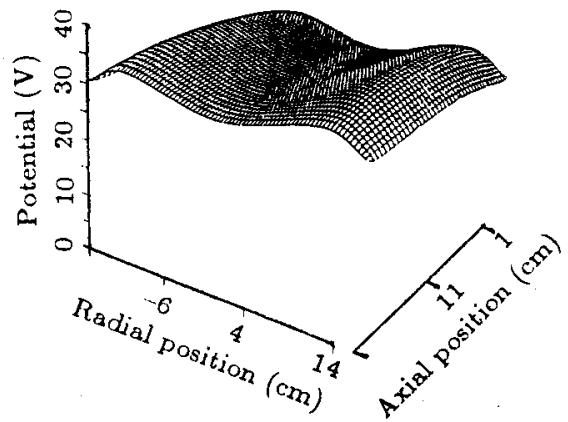

Fig. 8. Spatial distribution of plasma potential for case II.

obvious as that of the case II. The spatial distributions of plasma density for cases I and II are displayed by Figs. 5 and 6 , respectively. Similarly, case I density has its maximum value at the center and case II near the edgc area. This is because the plasma density distribution, with an assumption of uniform neutral gas density, is primarily dependent on the ionization rate which is a function of $T_{\mathrm{e}}$. The plasma potential distrbutions for cases I and II are shown by Figs. 7 and 8 , respectively. Analogously, there is one peak at the center of the plasma bulk for case I and two peaks near

the chamber edge for case II. Since the ion movement is mainly dominated by the plasma potential, the ion flux has the similar feature. Figures 9 and 10 are the ion flux profiles. It can be found that the flux along the bottom surface is peaked at the radial centerline for case I (see Fig. 9). However, the ion flux across the radius along the bottom goes up to the maximum at $r=11 \mathrm{~cm}$ for case II (see Fig. 10). Therefore, it is concluded that the plasma parameters, such as $T_{c}, n$ and $\phi$ can be partly controlled by the external applied coil configuration.

\section{REFERENCES}

[1] R. K. Porteous and D. B. Graves, IEEE tran on Plasma Science, 19 (1991) 204.

[2] M. Hussein and G. A. Emmert, J. Vac. Sci. Technol. 8 A (1990) 2913.

[3] Y.H. Lee, J.E. Heidenreich and G. Fortuno, J. Vac. Sci. Technol. A 7 (1989) 903.

[4] P. H. Singer, Semiconductor International, July, (1991) 46

[5] S. M. Gorbatkin, L. A. Berry and J. B. Roberto, J. Vac. Sci. Tech. A 8 (3) (1900) 2983.

[6] R. A. Stewart, X.Y. Qian, D. A. Carl, B. Lake, Jr., J. Benasso, R. Lynch, C. A. Pico, M. A. Liebermen and N.W. Cheung, Memorandum No. UCB/ERL M90/100, 12 Nov., 1990, Electronics Research Laboratory, College of Engineering, University of California at Berkeley, 94720.

[7] D. B. Graves, H-M Wu and R. K. Portcous, Jpn. J. Appl. Phys. 32, Part 1, 6 B (1993) 2990.

[8] R. K. Porteous, H-M Wu and D. B. Graves, Plasma Source and Technology, 3 (1094) 25. 